USTC-ICTS-03-06

\title{
REMARKS ON 't HOOFT'S BRICK WALL MODEL
}

\author{
Hua Bai* \\ Interdisciplinary Center for Theoretical Study, University of Science and Technology of China \\ Hefei, Anhui 230026, P.R. China \\ Mu-Lin $\operatorname{Yan}^{\dagger}$ \\ CCAST(World Lab), P.O. Box 8730, Beijing, 100080, P.R. China \\ and \\ Interdisciplinary Center for Theoretical Study, University of Science and Technology of China \\ Hefei, Anhui 230026, P.R. China
}

(October 29, 2018)

\begin{abstract}
A semi-classical reasoning leads to the non-commutativity of the space and time coordinates near the horizon of Schwarzschild black hole. This non-commutativity in turn provides a mechanism to interpret the brick wall thickness hypothesis in 't Hooft's brick wall model as well as the boundary condition imposed for the field considered. For concreteness, we consider a noncommutative scalar field model near the horizon and derive the effective metric via the equation of motion of noncommutative scalar field. This metric displays a new horizon in addition to the original one associated with the Schwarzschild black hole. The infinite red-shifting of the scalar field on the new horizon determines the range of the noncommutativ space and explains the relevant boundary condition for the field. This range enables us to calculate the entropy of black hole as proportional to the area of its original horizon along the same line as in 't Hooft's model, and the thickness of the brick wall is found to be proportional to the thermal average of the noncommutative space-time range. The Hawking temperature has been derived in this formalism. The study here represents an attempt to reveal some physics beyond the brick wall model.
\end{abstract}

The brick wall model proposed by 't Hooft has been used for the purpose of deriving the entropy of black hole and other quantities [1] [2], and has been extensively studied (an incomplete list, see Refs. [3] [4]). In the model, the thickness of the brick wall near the horizon of Schwarzschild black hole was set to be

$$
h=\frac{N^{\prime} l_{p}^{2}}{360 \pi r_{H}}
$$

where $r_{H}$ is the radius at which the horizon is located, $l_{p}=\sqrt{G}$ (in this letter $\hbar=c=1$ ) the Planck length and $N^{\prime}$ the number of the multiplet of the quantum field in the model. Eq.(1) is a prior hypothesis of the brick wall model. Actually, only under this hypothesis, the thermodynamic properties of a black hole can be reproduced correctly. Namely, this model can lead to the correct Bekenstein-Hawking entropy formula

$$
S_{B H}=\frac{1}{4} \frac{A}{G}
$$

where $S_{B H}$ is Bekenstein-Hawking entropy [5] [6] and $A$ is the horizon area . In this letter, we try to derive the brick wall thickness by a semi-classical argument and to reveal some underlying physics related to this hypothesis.

For the sake of definiteness, we study the $3+1$ dimensional Schwarzschild black hole. In this case, $\partial_{t}$ is the time Killing vector. Its global energy (or the mass of the black hole) is $E_{B H}=M=r_{H} / 2 G$. Rather than thinking black hole as a classical object, we treat it as a quantum state with high degeneracy and its degrees of freedom are located near by the horizon. Treating the energy $E_{B H}$ and its conjugate time coordinate as operators, quantum mechanics tells that these two quantities can not be measured simultaneously. In other words, $E_{B H}$ and t satisfy Heisenberg

\footnotetext{
*E-mail address: huabai@mail.ustc.edu.cn

${ }^{\dagger}$ E-mail address: mlyan@staff.ustc.edu.cn

${ }^{\ddagger}$ mail address
} 
uncertainty relation $[t, E]=i$. Given the relation $E_{B H}=r_{H} / 2 G$, we must conclude that the uncertainty of $E_{B H}$ imply that of $r_{H}$, and then we have

$$
\left.[t, r]\right|_{r=r_{H}}=i 2 l_{p}^{2},
$$

This equation implies that the radial coordinate is noncommutative with the time at the horizon. The corresponding uncertainty relation for them is $\left.(\Delta t)(\Delta r)\right|_{r \sim r_{H}} \sim 2 l_{p}^{2}$. In other words, due to quantum measurement effects, $r$ spreads in the range of $\left(r-\Delta r, r_{H}+\Delta r\right)$. Namely, eq.(3) is extended as follows

$$
\left.[t, r]\right|_{r \in\left(r_{H}-\Delta, r_{H}+\Delta\right)}=i 2 l_{p}^{2} .
$$

where $\Delta$ is a distance about Planck length scale. Here, for simplicity, we have denoted on $\Delta r=\Delta$ with $\Delta$ on the order of $l_{p}$. For a classical observer at infinity, he can only detect events happening at $r>r_{H}$. For this reason, we only need to consider $r>r_{H}$ at least semi-classically. In the range of $\left(r_{H}, r_{H}+\Delta\right)$, the classical $\phi^{a}$-fields are noncommutative. The non-commutative theory in field theory and in string theory has been discussed recently in $[7,8]$. As $r>r_{H}+\Delta$, the fields are commutative as usual since

$$
\left.[t, r]\right|_{r>r_{H}+\Delta}=0 .
$$

According to our aforementioned discussion, we should construct a brick wall model with noncommutative $\phi^{a}$-fields in the range of $\left(r_{H}, r_{H}+\Delta\right)$ and with commutative $\phi^{a}$-fields in $\left(r_{H}+\Delta, L\right)$ where $L$ represents an infrared cutoff in the model. It is essential that $\Delta$ should be an intrinsic quantity of the model, which characterizes the boundary between the noncommutative space-time range and the commutative space-time range, and should be determined by the model itself consistently. Surprisingly, this expectation can be realized and a brick wall model without the brick wall thickness hypothesis can be constructed by the following considerations: 1) Starting with a simplest noncommutative $\phi^{a}$-field action within metric of Schwarzschild black hole, the equation of motion of $\phi^{a}$ can be derived exactly; 2) This equation of motion in noncommutative field theory should be, of course, quite different from the ordinary KleinGordon equation of $\phi^{a}$-fields within Schwarzschild metric. This fact implies $\phi^{a}$-fields should be moved in a curve space with a new effective metric $\widetilde{g}^{\mu \nu}$ for a classical observer at infinity; 3) Remarkably, it will be shown below that $\widetilde{g}^{t t}$ has two new singularities besides the original one at $r=r_{H}$ : one is outside the horizon of the black hole and another is in inside. Denoting their locations as $r_{H} \pm \Delta^{\prime}$ respectively, we will find $\Delta^{\prime}$ is dependent on the energies of the noncommutative fields $E$, i.e., $\Delta^{\prime}=\Delta^{\prime}(E)$. This means that to the fields with energy $E, \phi_{E}^{a}(r)$, its red-shifting on the $\left(r_{H} \pm \Delta^{\prime}(E)\right)$-surface is infinite due to $\widetilde{g}_{00}\left(r=r_{H}+\Delta(E)\right)=0$, and then we have

$$
\left.\phi_{E}^{a}(r)\right|_{r=r_{H}+\Delta^{\prime}(E)}=0 ;
$$

4) We argue that the fact that the noncommutative fields vanish at $r=r_{H}+\Delta^{\prime}(E)$ means we can think the space-time coordinates on the surface of $r=r_{H}+\Delta^{\prime}(E)$ to be commutative, i.e., $\left.[t, r]\right|_{r=r_{H}+\Delta^{\prime}(E)}=0$. And then we will further have

$$
\left.[t, r]\right|_{r \geq r_{H}+\Delta^{\prime}(E)}=0 .
$$

Comparing eq. (7) with (5), we get

$$
\Delta^{\prime}(E)=\Delta .
$$

Namely, $\Delta=\Delta(E)$ can be determined by using eqs. (6) and (8), and the ordinary brick wall model works in the raging of $\left(r_{H}+\Delta, L\right)$, whose ultraviolet boundary condition is eq. (6). Consequently, we have constructed a new model without the brick wall thickness hypothesis by starting with thinking the black hole as a quantum state with high degeneracy. In the follows, we shall apply this model to derive the entropy of the Schwarzschild black hole, and to interpret 't Hooft's hypotheses on the brick wall thickness.

With the above, we now consider the classical field as a probe in the region of $r_{H}<r<r_{H}+\Delta(E)$ but moving in a non-commutative background. We rewrite eq. (4) as follows

$$
\begin{aligned}
& {\left[x^{i}, x^{j}\right]=i \Theta \varepsilon^{i j}, \quad(i, j=0,1), \quad\left(x^{0}=t, x^{1}=r\right), \quad \Theta=2 l_{p}^{2},} \\
& {\left[x^{k}, x^{\mu}\right]=0, \quad(k=2,3 ; \mu=0,1,2,3)}
\end{aligned}
$$

where $\varepsilon^{i j}$ is an antisymmetrical tensor with $\varepsilon^{01}=1$. The star product of two function $f(x)$ and $g(x)$ is given by the Moyal formula: 


$$
(f \star g)(x)=\left.\exp \left[\frac{i}{2} \Theta \varepsilon^{i j} \frac{\partial}{\partial x^{i}} \frac{\partial}{\partial y^{j}}\right] f(x) g(y)\right|_{y=x} .
$$

For simplicity, we consider only the coupling between the field and the background, i.e.,

$$
I=-\frac{1}{2} \int d^{4} x \sqrt{-g} g^{\mu \nu}\left(\partial_{\mu} \phi_{E} \star \partial_{\nu} \phi_{E}\right) \quad\left(r_{H}<r<r_{H}+\Delta\right)
$$

where $g_{\mu \nu}$ is the metric of a Schwarzschild black hole,

$$
d s^{2}=-\left(1-\frac{r_{H}}{r}\right) d t^{2}+\left(1-\frac{r_{H}}{r}\right)^{-1} d r^{2}+r^{2}\left(d \theta^{2}+\sin \theta^{2} d \varphi^{2}\right) .
$$

We evaluate the start product in the action using Eq. (11) and cast the action in the ordinary product. By this, the noncommutative effect can be absorbed into an equivalent background metric. In other words, we first take the semi-classical quantum effect into consideration. This effect is then realized through the non-commutative geometry. Finally, this effect is further through an effective background but in an ordinary geometry. For a given energy mode, i.e., assuming $\phi_{E}(t, r, \theta, \varphi)=e^{-i t E} f(r, \theta, \varphi)$, the effective metric can be either read from the action (actually simpler) or from the following equation of motion for the scalar field once the star product is evaluated:

$$
\begin{aligned}
& \partial_{t}\left(\sqrt{-g} g^{t t} \partial_{t} \phi^{a}\right)+\partial_{r}\left(\sqrt{-g} g^{r r} \partial_{r} \phi^{a}\right)+\partial_{\theta}\left(\sqrt{-g} g^{\theta \theta} \partial_{\theta} \phi^{a}\right)+\partial_{\varphi}\left(\sqrt{-g} g^{\varphi \varphi} \partial_{\varphi} \phi^{a}\right)+ \\
& \frac{1}{2 !}\left(\frac{i \Theta}{2}\right)^{2}\left(\sqrt{-g} g^{r r}\right)_{, r r} \partial_{t} \partial_{t} \partial_{r} \partial_{r} \phi^{a}+\sum_{n=1}^{\infty} \frac{1}{2 n !}\left(\frac{i \Theta}{2}\right)^{2 n}\left(\sqrt{-g} g^{t t}\right), \underbrace{r \cdots r}_{2 n} \underbrace{\partial_{t} \cdots \partial_{t}}_{2 n+2} \phi^{a}=0
\end{aligned}
$$

where $\left(\sqrt{-g} g^{t t}\right), \underbrace{r \cdots r}_{n}$ stands for $\underbrace{\partial_{r} \cdots \partial_{r}}_{n}\left(\sqrt{-g} g^{t t}\right)$, etc. For the scalar field with given energy $E$, the above equation becomes

$$
\begin{aligned}
& {\left[-\frac{\sin \theta r^{3}}{r-r_{H}}-\frac{\sin \theta \Theta^{2} E^{2}}{4}-\frac{\sin \theta r_{H}^{3}}{r-r_{H}} \sum_{n=1}^{\infty}\left(\frac{\Theta E}{2\left(r-r_{H}\right)}\right)^{2 n}\right] \phi_{E, t t}^{a}} \\
& +\left[\sin \theta r\left(r-r_{H}\right)+\frac{\sin \theta \Theta^{2} E^{2}}{4}\right] \phi_{E}^{a}, r r+\partial_{\theta}\left(\sin \theta \partial_{\theta} \phi^{a}\right)+\frac{1}{\sin \theta} \phi_{E}^{a}, \varphi \varphi=0
\end{aligned}
$$

Noticing

$$
\sum_{n=1}^{\infty}\left(\frac{\Theta E}{2\left(r-r_{H}\right)}\right)^{2 n}=\frac{1}{1-\frac{\Theta^{2} E^{2}}{4\left(r-r_{H}\right)^{2}}}-1
$$

we can read the effective metric $\tilde{g}_{\mu \nu}$ from Eq. (15) as

$$
\begin{aligned}
& -\frac{\sin \theta r^{3}}{r-r_{H}}\left[1+\frac{\Theta^{2} E^{2}\left(r-r_{H}\right)}{4 r^{3}}+\frac{r_{H}^{3}}{r^{3}}\left(\frac{1}{1-\frac{\Theta^{2} E^{2}}{4\left(r-r_{H}\right)^{2}}}-1\right)\right]=\sqrt{-\widetilde{g}}{ }^{t t} \\
& \sin \theta\left[\left[r\left(r-r_{H}\right)+\frac{\Theta^{2} E^{2}}{4}\right]=\sqrt{-\widetilde{g}} \widetilde{g}^{r r}\right. \\
& \sin \theta=\sqrt{-\widetilde{g}} \widetilde{g}^{\theta \theta} \\
& \frac{1}{\sin \theta}=\sqrt{-\widetilde{g}} \widetilde{g}^{\varphi \varphi}
\end{aligned}
$$

From the above, we can solve the effective metric as

$$
\begin{aligned}
& \sin \theta^{2} \widetilde{g}_{\theta \theta}=\widetilde{g}_{\varphi \varphi}, \\
& \widetilde{g}_{t t} \widetilde{g}_{r r}=-1, \\
& \widetilde{g}_{t t}=-\left(1-\frac{r_{H}}{r}\right) \sqrt{\frac{1+\frac{\Delta^{2}(E)}{r\left(r-r_{H}\right)}}{1+\frac{\Delta^{2}(E)\left(r-r_{H}\right)}{r^{3}}+\frac{r_{H}^{3}}{r^{3}}\left[\frac{1}{1-\frac{\Delta^{2}(E)}{\left(r-r_{H}\right)^{2}}}-1\right]},} \\
& \tilde{g}_{\theta \theta}^{2}=\left[r\left(r-r_{H}\right)+\Delta^{2}(E)\right]\left[r^{2}+r r_{H}+r_{H}^{2}+\Delta^{2}(E)+\frac{\left(r-r_{H}\right) r_{H}^{3}}{\left(r-r_{H}\right)^{2}-\Delta^{2}(E)}\right],
\end{aligned}
$$


where we have set $\Delta(E)=\Theta E / 2$. This effective metric is quite different from the original one. The semi-classical effect causes the unexpected appearance of a new horizon at $r=r_{H}+\Delta(E)$ at which $\tilde{g}_{t t}$ vanishes. Note also that the $\tilde{g}_{\theta \theta}$ blows up at this point which implies that the curvature scalar vanishes at this point, too. therefore a regular one. Note that, as also discussed in [2], the energy $E$ for the scalar $\phi$ shouldn't be too large, therefore $\Delta(E)=\Theta E / 2$ is not larger than the Planck length.

With the above, it is now not difficult to understand the boundary condition (6) (with (8)) we given earlier. Because of the appearance of the new horizon, an observer cannot detect the $\phi_{E}$ at $r \leq r_{H}+\Delta(E)$. Just we have discussed earlier that the field has an infinite red-shift at $r=r_{H}+\Delta(E)$. And since the noncommutative property of $\phi_{E}^{a}$ is caused by $[t, r] \neq 0, \phi_{E}^{a}\left(r_{H}+\Delta(E)\right)=0$ means $[t, r]=0$ at $r=r_{H}+\Delta(E)$, as $r>r_{H}+\Delta(E)$, the field $\phi_{E}$ should be a commutative one as discussed earlier. This in turn provides an explanation for the short-distance cutoff, or the brick wall thickness $h$, introduced in 't Hooft's model. In other words, the $h$ has its origin of our $\Delta(E)$. We emphasize again that there is no brick wall in our model, and the $\Delta(E)$ is derived by the action (12). With this understanding, we can now follow 't Hooft's method [2] to evaluate the black hole entropy in our model. For this purpose, we consider $N$ scalar fields each with energy $E$ in the original Schwarzschild black hole background. The action in the range of $\left(L>r>r_{H}+\Delta(E)\right)$ reads

$$
I=-\frac{1}{2} \int d^{4} x \sqrt{-g} g^{\mu \nu}\left(\partial_{\mu} \phi_{E}^{a} \partial_{\nu} \phi_{E}^{a}\right), \quad\left(r>r_{H}+\Delta(E)\right) .
$$

The eq. (6) serves as the ultraviolet boundary condition of $\phi_{E}^{a}$. And according to refs $[1,2]$ another boundary condition can be taken as $\phi_{E}^{a}(r=L)=0$, where $L$ is large.

Using the $W K B$ approximation and by setting $\phi_{E}^{a}=e^{-i E t-i \int k(r) d r} Y_{l m}(\theta, \varphi)$, we have the radial wave number $k(r, l, E)$ from the corresponding equation of motion:

$$
k^{2}=\left(1-\frac{r_{H}}{r}\right)^{-1}\left[\left(1-\frac{r_{H}}{r}\right)^{-1} E^{2}-r^{-2} l(l+1)\right], \quad \text { in the range of }\left(L>r>r_{H}+\Delta(E)\right) .
$$

The number of states below energy $E$ is

$$
\begin{aligned}
g(E) & =N \int d l(2 l+1) \int_{r_{H}+\Delta(E)}^{L} d r \sqrt{k^{2}(l, E)} \\
& =\frac{2 N}{3} \int_{r_{H}+\Delta(E)}^{L} d r \frac{r^{4} E^{3}}{\left(r-r_{H}\right)^{2}} .
\end{aligned}
$$

Note that be different from 't Hooft's brick wall model, there is no an additional ultraviolet cutoff to be introduced in above calculation of $g(E)$. The free energy then reads

$$
\begin{aligned}
\pi \beta F & =\int d g(E) \ln \left(1-e^{-\beta E}\right) \\
& =-\int_{0}^{\infty} d E \frac{\beta g(E)}{e^{\beta E}-1} \\
& =-\frac{2 N}{3} \int_{0}^{\infty} d E \frac{\beta E^{3}}{e^{\beta E}-1} \int_{r_{H}+\Delta(E)}^{L} d r \frac{r^{4}}{\left(r-r_{H}\right)^{2}}
\end{aligned}
$$

The dominant contribution from the event horizon to $F$ is

$$
F \approx-\frac{8 N \zeta(3) r_{H}^{4}}{3 \pi \Theta \beta^{3}}
$$

where we have used $\Delta(E)=\Theta E / 2$ and $\zeta(3)$ is Riemann $\zeta$-function, $\zeta(3)=\sum_{n=1}^{\infty} 1 / n^{3} \approx 1.202$.

The entropy of the black hole can now be obtained as

$$
S=\beta^{2} \frac{\partial F}{\partial \beta}=\frac{8 N \zeta(3) r_{H}^{4}}{\pi \Theta \beta^{2}}
$$

Now, let us derive the inverse temperature $\beta$ in the above equation in the formalism of this present paper. There are great deal of works in the literature to study and to discuss the black hole's temperature $\beta^{-1}$ (e.g., see [6] [9]- [15]). We employ the methods of Damour-Ruffini [9] and of Sannan [10] to do so. In this method, the point is that we should 
derive the outgoing wave functions of $\phi_{E}$ in both outside and inside of the black hole. Using the $\phi_{E}$-wave-number expression (26), the incoming wave function of $\phi_{E}$ reads

$$
\phi_{E}^{i n}=\exp \left[-i E t-i \int k(r) d r\right]=\exp \left[-i E\left(t+\int_{c}^{r} \frac{r}{\left(r-r_{H}\right)} d r\right)\right] .
$$

In the above expression, the integration $\int_{c}^{r} r /\left(r-r_{H}\right) d r=r-c+r_{H} \ln \left(\left(r-r_{H}\right) /\left(c-r_{H}\right)\right)$ is actually of the so called tortoise coordinate $r^{*}=r+r_{H} \ln \left(\left(r-r_{H}\right) / r_{H}\right)$ used in ref. [9], expect an unimportant constant. For simplicity, we take $c=r_{H}+\Delta(E)$, and set $t+\int_{r_{H}+\Delta(E)}^{r} r /\left(r-r_{H}\right) d r=v$ which is the usual advanced Eddingtion-Finkelstein coordinates. Then the incoming wave function is $\phi_{E}^{i n}=e^{-i E v}$, and the outgoing wave function in the range $r>r_{H}+\Delta(E)$ is

$$
\phi_{E}^{\text {out }}\left(r>r_{H}+\Delta(E)\right)=A_{E} \exp \left[-i E t+i \int_{r_{H}+\Delta(E)}^{r} \frac{E r}{\left(r-r_{H}\right)} d r\right]=A_{E} \phi_{E}^{i n} \exp \left[2 i \int_{r_{H}+\Delta(E)}^{r} \frac{E r}{\left(r-r_{H}\right)} d r\right]
$$

where $A_{E}$ is normalized constant. From the equation(15) which holds in the range of $r_{H}-\Delta(E)<r<r_{H}+\Delta(E)$ (see eq.(4)), the square of $\phi_{E}$-wave- number $k^{\prime 2}(r)$ reads

$$
k^{\prime}(r)^{2}=\frac{E^{2} r^{2}}{\left(r-r_{H}\right)^{2}+\frac{\left(r-r_{H}\right) \Delta^{2}(E)}{r}}\left[1+\frac{\left(r-r_{H}\right) \Delta^{2}(E)}{r^{3}}+\frac{r_{H}^{3}}{r^{3}}\left(\frac{\left(r-r_{H}\right)^{2}}{\left(r-r_{H}\right)^{2}-\Delta^{2}(E)}-1\right)-\frac{l(l+1)\left(r-r_{H}\right)}{E^{2} r^{3}}\right] .
$$

By using S-wave approximate, the wave number $k^{\prime}$ reduces to be

$$
k^{\prime}(r)= \pm i \frac{E r_{H}}{\Delta(E) \sqrt{1-x^{2}}} \sqrt{\frac{x+3 \xi\left(x^{2}-1\right)+3 \xi^{2} x\left(x^{2}-1\right)+\xi^{3}\left(x^{4}-1\right)}{x(1+x \xi)\left(1+\frac{\xi}{\xi x^{2}+x}\right)}}
$$

where $x=\left(r-r_{H}\right) / \Delta(E)$ and $\xi=\Delta(E) / r_{H}$. Note, the mass of the black hole we concern is much larger than the Planck mass, hence $\xi \ll 1$. Considering the continuity of wave functions of $\phi_{E}^{\text {out }}$, and using eq.(32), the outgoing wave function in the inside of the black hole, $\phi_{E}^{\text {out }}\left(r<r_{H}-\Delta(E)\right)$, is obtained as follows

$$
\phi_{E}^{\text {out }}\left(r<r_{H}-\Delta(E)\right)=A_{E} \phi_{E}^{i n} \exp \left[2 i \int_{r_{H}+\Delta(E)}^{r_{H}-\Delta(E)} k^{\prime}(r) d r\right] \exp \left[2 i \int_{r_{H}-\Delta(E)}^{r} \frac{E r}{\left(r-r_{H}\right)} d r\right] .
$$

Using eq.(34), the first integration in RHS of above equation can be calculated,

$$
2 i \int_{r_{H}+\Delta(E)}^{r_{H}-\Delta(E)} k^{\prime}(r) d r= \pm 2 E r_{H} \int_{-1}^{1} \frac{d x}{\sqrt{1-x^{2}}} \sqrt{\frac{x+3 \xi\left(x^{2}-1\right)+3 \xi^{2} x\left(x^{2}-1\right)+\xi^{3}\left(x^{4}-1\right)}{x(1+x \xi)\left(1+\frac{\xi}{\xi x^{2}+x}\right)}} .
$$

Since $\xi \ll 1$, we have

$$
2 i \int_{r_{H}+\Delta(E)}^{r_{H}-\Delta(E)} k^{\prime}(r) d r \simeq \pm\left(2 \pi r_{H} E \pm i 2 \pi r_{H} E \xi+3 \pi r_{H} E \xi^{2}+O\left(\xi^{3}\right)\right) \simeq \pm 2 \pi r_{H} E(1 \pm i \xi) .
$$

where the terms of $O\left(\xi^{2}\right)$ have been neglected (due to $\xi \ll 1$ ), whose effects will be briefly discussed in the end of this paper. Taking the positive sign, we obtain the absolute value of ratio of the outgoing wave function's amplitude outside the black hole to the one inside the black hole as follows

$$
\left|\frac{\phi_{E}^{\text {out }}\left(r>r_{H}+\Delta(E)\right)}{\phi_{E}^{\text {out }}\left(r<r_{H}-\Delta(E)\right)}\right|=e^{-2 \pi r_{H} E}
$$

According to the Sannan's discussions [10] and using eq.(38), the relative scattering probability from the event horizon reads

$$
P_{E}=e^{-4 \pi r_{H} E} .
$$

This means that the $\phi_{E}$-particle mean number $\left\langle\mathcal{N}_{E}\right\rangle$ in the radiation is as follows [10]

$$
\left\langle\mathcal{N}_{E}\right\rangle=\frac{\left|\Gamma_{E}\right|^{2}}{e^{4 \pi r_{H} E}-1} \equiv \frac{\left|\Gamma_{E}\right|^{2}}{e^{E / T_{H}}-1}
$$


where $\left|\Gamma_{E}\right|^{2}$ is the frequency-dependent transmission coefficient for the outgoing wave to reach future infinity. Consequently, in the formalism of this present paper we obtain

$$
\beta^{-1}=T_{H}=\frac{1}{4 \pi r_{H}}
$$

which is same as the Hawking temperature.

With the above, let's return to the deriving of the black hole entropy. Substituting eq.(41) into Eq.(30), we get the entropy of the black hole as follows

$$
S=\frac{\zeta(3) N}{16 \pi^{4}} \frac{A}{G}
$$

where $A=4 \pi r_{H}^{2}$ is the horizon area. So we reproduce the correct relation of $S \propto A$, which implies that we are on the right track. We shouldn't expect that the precise Bekenstein-Hawking entropy can be obtained via the brick-wall model even with our semi-classical consideration without fine tuning certain parameters. Nevertheless, our semiclassical consideration does provide explanations to the boundary condition imposed for the field considered and to the brick wall thickness parameter $h$, therefore, still quite remarkable.

If we set the number of the quantum field multiplet $N=4 \pi^{4} / \zeta(3) \approx 324$, then Eq. (42) does give the right entropy. The statistical average value of $\Delta(E)$ can be obtained as

$$
\overline{\Delta(E)}=l_{p}^{2} \frac{\int_{0}^{\infty} \frac{E d g(E)}{e^{\beta E}-1}}{\int_{0}^{\infty} \frac{d g(E)}{e^{\beta E}-1}} \approx \frac{3 \zeta(3) l_{p}^{2}}{\pi^{3} r_{H}}
$$

The brick wall thickness given in Eq.(1) can be related to the above $\overline{\Delta(E)}$ via

$$
h=\eta \overline{\Delta(E)}, \quad \eta=\frac{N^{\prime} \pi^{2}}{1080 \zeta(3)} .
$$

This indicates that the thickness hypothesis in the brick wall model reflects the average effect of the noncommutative field theory in the wall. Since the non-commutativity arises from the semi-classical consideration, therefore we provide a direct link of the thickness hypothesis in the model to the underlying quantum effect as expected.

Finally, we argue that the effects of $O\left(\xi^{2}\right)$ in eq.(36) raise the effective temperature of the hole as it radiates. Namely, the $\xi$-dependency in the eq.(36) should be thought as its thermal statistical average $\bar{\xi}$-dependency. Like eq.(43), $\overline{\xi^{2}}=l_{p}^{4} \overline{E^{2}} / r_{H}^{2}=1 / 40 \times\left(l_{p} / r_{H}\right)^{4}$, then the effective temperature for the hole is

$$
T_{\text {eff }}=T_{H}\left(1-\frac{3 l_{p}^{4}}{80 r_{H}^{4}}\right) .
$$

where the $T_{H}$ is Hawking temperature and the second term in the right-hand-side represents a correction to the temperature due to the space-time non-commutative property near the event horizon eq.(4). Obviously, this correction to the $T_{H}$ is tiny as $r_{H}>>l_{p}$, and hence it can be ignored indeed. The corrections of $O\left(\xi^{N}\right)$ with $N>2$ can be analyzed likewise, and they are also ignorable as $r_{H}>>l_{p}$.

\section{ACKNOWLEDGMENTS}

The authors wish to thank Jiliang Jing, Jian-Xin Lu and Shuang-Qing Wu for their stimulating discussions. We would like also to thank the referee for his important suggestion on deriving the black hole temperature in this paper. This work is partially supported by NSF of China 90103002 and the Grant of the Chinese Academy of Sciences.

[1] G.'t Hooft, Nucl. Phys. B256 (1985) 727.

[2] G. 't Hooft, Int.J.Mod.Phys. A11 (1996) 4623-4688. 
[3] R.B. Mann, L. Tarasov and A. Zelnikov, Class. Quant. Grv.9, (1992)1487. J.-G. Demers,R.Lafrance and R.C.Myers,Phys.Rev.D52,(1995)2245[gr-qc/9503003]. A.Ghosh and P.Mitra,Phys.Lett.B357,(1995)295[hep-th/9411128]. V.P. Frolov, D.V. Fursaev and A.I. Zelnikov, Phys.RevD 54,(1996)2711[hep-th/9512184];Phys.Lett. 382,(1996)220[hepth/9603175]; V.P. Frolov and D.V. Fursaev, Class.Quant.Grav. 15,(1998)2041[hep-th/9802010]. S.N.Solodukhin,Phys.Rev.D54,(1996)3900[hep-th/9601154];Phys.Rev.D56,(1997)4968[hep-th/9612061]. S.-W. Kim, W.T. Kim, Y.-J. Park and H. Shin, Class.Quant.Grav. 14,(1997)2617[gr-qc/9704032]. S.P. Kim, S.K. Kim, K.-S Soh and J.H. Yee, Phys.Rev. D55,(1997)2159[gr-qc/9608015]. G. Cognola and P. Lecca, Phys.Rev D57,(1998)1108[hep-th/9706065]. E. Abdalla and L.A. Correa-Borbonet, Mod.Phys.Lett. A16,(2001)2495[hep-th/0012101]. S. Mukohyama and W. Israel, Phys.Rev. D58,(1998)104005[gr-qc/9806012]. E. Winstanley, Phys.Rev. D63,(2001)084013[hep-th/0011176].

[4] J. Jing and M.L. Yan. Phys.Rev. D64,(2001)064015; ibid, D63,(2001)084028; ibid, D61,(2000)044016; ibid, D60,(1999)084015.

[5] J. D. Bekenstein, Lett. Nuovo Cimento 4, 737 (1972); Phys. Rev. D 7(1973) 2333

[6] S. W. Hawking, Nature (London) 248, 30 (1974); Commun. Math. Phys. 43(1975)199 ; Phys Rev . D14 (1976) 2460; The unpredictability of quantum gravity, Cambridge Univ, preprint(May 1982)

[7] N.Seigerg and E.Witten, JHEP.9909 (1999) 032.

[8] S.Minwalla,M.V. Raamsdonk and N. Seiberg, JHEP.0002(2000) 020

[9] T. Damour, R. Ruffini, Phys Rev.D14(1976) 332

[10] S. Sannan, Gen. Rel. Grav.20 (1988) 239

[11] J.B. Hartle, S.W. Hawking. Phys. Rev. D13 (1976) 2188

[12] G.W. Gibbons, M.J. Perry. Proc. R. Soc. Lond, A358 (1978) 467

[13] W.G. Unruh. Phys. Rev. D14 (1976) 870

[14] see also: N.D. Birrell. P.C.W. Davies. Quantum Fields in Curved Space. Cambridge: Cambridge University Press, 1982.

[15] M.K. Parikh, F. Wilczek. Phys. Rev. Lett. 85 (2000) 5042 